\title{
A COMBINATORIAL COINCIDENCE PROBLEM
}

\author{
BY Z. A. MELZAK ${ }^{1}$
}

Communicated by N. Levinson, July 7, 1967

Let $A \subset E^{m}(m \geqq 1)$, let $B(o) \subset E^{m}$ be convex with center of symmetry at $o$, let $n$ and $p$ be integers $(1 \leqq p \leqq n, n \geqq 2)$, and let $f(u)$ be an integrable function defined on $A$. Let $A^{n}$ be the Cartesian product of $A$ with itself $n$ times and define $Y \subset A^{n}$ by

$$
\begin{aligned}
Y=\left\{x=\left(x_{1}, \cdots, x_{n}\right): \bigcap_{k=1}^{p} B\left(x_{i_{k}}\right) \neq \varnothing\right. & \\
& \left.\quad \text { for some } i_{1}, \cdots, i_{p}, 1 \leqq i_{1}<\cdots<i_{p} \leqq n\right\} .
\end{aligned}
$$

The problem of evaluating $J=\int_{Y} \prod_{1}^{n} f\left(x_{i}\right) d x_{1} \cdots d x_{n}$ generalizes a number of questions in probability, queuing theory, scattering, statistical mechanics etc., [1], [2]. Put

$$
\begin{aligned}
M=\left(\begin{array}{l}
n \\
p
\end{array}\right), S_{i_{1} \cdots i_{p}} & =\left\{\left(x_{1}, \cdots, x_{n}\right): \bigcap_{s=1}^{p} B\left(x_{i_{s}}\right) \neq \varnothing\right\}, F(x) \\
& =\prod_{1}^{n} f\left(x_{i}\right), d V=d x_{1} \cdots d x_{n}
\end{aligned}
$$

and let the $M$ sets $S_{i_{1} \cdots i_{p}}$ be enumerated as $\left\{S_{k}\right\}, k=1, \cdots, M$. Then by the inclusion-exclusion principle [2]

$$
\begin{aligned}
J & =\sum_{r=1}^{n}(-1)^{r+1}\left[\sum_{1 \leq k_{1}<\cdots<k_{r} \leqq M} \int_{S_{k_{1}} \cap \cdots n S_{k_{r}}} F(x) d V\right] \\
& =\sum_{r=1}^{n}(-1)^{r+1} U_{r},
\end{aligned}
$$

say. To help us keep track of different $r$-tuples of $p$-tuples, we introduce a generalization of graphs. Let $X$ be a regular simplex in $E^{n-1}$ with the vertices $w_{1}, \cdots, w_{n}$, a ( $d$-dimensional) hypergraph $G$ on $X$ is just a collection of some of the $\left(C_{d+1}^{n}\right) d$-dimensional faces of $X$; the number of vertices of $X$ lying in $G$ will be denoted by $v(G) . G$ is called a $(B, r)$-hypergraph on $X$ if it consists of $r$ such $d$-faces and if there are some $v=v(G)$ translates $B_{1}, \cdots, B_{v}$ of $B$ such that any $d+1$ of them, say $B_{1}, \cdots, B_{d+1}$, intersect if the corresponding vertices $w_{1}, \cdots, w_{d+1}$ lie in a $d$-face of $X$ included in $G$.

${ }^{1}$ Permanent address: Dept. of Mathematics, University of British Columbia. 
$G$ is called connected if no hyperplane in $E^{n-1}$ strictly separates some of its $d$-faces from the rest of them. Let $t=t(r, d)$ be the number of types of (topologically) distinct $(B, r)$-hypergraphs on $X$, let $G_{j}$ be any one of the $j$ th type, and let $M_{r j}^{d}(n)$ be the number of distinct $(B, r)$-hypergraphs on $X$ of the $j$ th type. Let $J_{0}=\int_{A} f(u) d u$, if $d=p-1$ observe that each $d$-face of a $(B, r)$-hypergraph corresponds to exactly one set $S_{k}$; let

$$
J(G)=\int_{S_{k_{1}} \cap \ldots \cap} \int_{S_{k_{r}}} F(x) d V
$$

where $S_{k_{1}}, \cdots, S_{k_{r}}$ are the $S$-sets corresponding to the $d$-faces of $G$. Now we get a formula for the summand $U_{r}$ of (1):

$$
U_{r}=\sum_{j=1}^{t(r, p-1)} M_{r j}^{p-1}(n) J_{0}^{n-v\left(G_{j}\right)} \prod_{C\left(G_{j}\right)} J\left(C\left(G_{j}\right)\right)
$$

where the product is taken over the connected components $C\left(G_{j}\right)$ of $G_{j}$. This generalizes some of the so-called cluster expansions of statistical mechanics [3].

In most applications it is found that $A$ and $B$ are simple regular sets (cubes, balls), $B$ is small while $A$ is large, and $f$ is well behaved. (1) and (2) allow us then, in principle at least, to expand $J$ in the powers of a parameter measuring the ratio of sizes of $B$ to $A$, and to estimate the error of truncation. The integrals $J\left(C\left(G_{j}\right)\right)$ can rarely be found analytically but the Monte-Carlo method lends itself very well to their numerical evaluation.

The following expansions and identities for iterated binomial coefficients were found in the process of evaluating the numbers $M_{r j}^{p-1}(n)$ in (2). Let $q=q(r, d)$ be the smallest integer $\geqq$ the largest positive root of $r=C_{x, d+1}$, then

$$
\left(\begin{array}{c}
n \\
d
\end{array}\right)=\sum_{k=q}^{r d} A_{k r}(d)\left(\begin{array}{l}
n \\
k
\end{array}\right)
$$

where

$$
\left.A_{k r}(d)=\sum_{j=0}^{k-q}(-1)^{j}\left(\begin{array}{l}
k \\
j
\end{array}\right)\left(\begin{array}{c}
k-j \\
d
\end{array}\right)\right) .
$$

Equating the coefficients of like powers of $n$ in (3) one gets 


$$
\begin{aligned}
& \sum_{j=0}^{d r-q}(-1)^{j}\left(\begin{array}{c}
d r \\
j
\end{array}\right)\left(\left(\begin{array}{c}
d r-j \\
d
\end{array}\right)\right)=(d r) ! /\left[r !(d !)^{r}\right], \\
& \sum_{j=0}^{d r-q-1}(-1)^{j}\left(\begin{array}{c}
d r-1 \\
j
\end{array}\right)\left(\left(\begin{array}{c}
d r-j-1 \\
d \\
r
\end{array}\right)\right)=d(d r) !(r-1) / 2\left[r !(d !)^{r}\right], \text { etc. }
\end{aligned}
$$

Details of proofs, computations, and applications will appear elsewhere.

\section{REFERENCES}

1. Z. A. Melzak, Scattering from random arrays, Quart. Appl. Math. 20 (1962), 151-159.

2. J. Riordan, Combinatorial analysis, Wiley, New York, 1958.

3. G. Uhlenbeck and G. W. Ford, Lectures in statistical mechanics, Lectures in Applied Math., Vol. I, Amer. Math. Soc., Providence, R. I., 1963.

Bell Telephone laboratories, Inc., Murray Hill, New Jersey

\section{FIXED POINTS OF NONEXPANDING MAPS}

\section{BY BEN JAMIN HALPERN}

Communicated by F. Browder, July 12, 1967

Introduction. This paper is concerned with nonexpanding maps from the unit ball of a real Hilbert space into itself. Browder [1] has established that such maps always possess at least one fixed point. We shall develop a method, which resembles the simple iterative method, for approximating fixed points of such maps. In fact, we shall generate a sequence, $\left\{x_{n}\right\}$, by the recursive formula $x_{n+1}$ $=k_{n+1} f\left(x_{n}\right)$ where $f$ is the map in question and $\left\{k_{n}\right\}$ is a sequence of real numbers. Our main result is Theorem 3 which states sufficient conditions on $k_{n}$ to insure the strong convergence of $x_{n}$ to a fixed point of $f$.

Definitions and preliminary observations. Let $H$ be a Hilbert space with inner product denoted by $($,$) and norm by \|\|$. Let $B$ be the unit ball, $B=\{x \in H \mid\|x\| \leqq 1\}$. A map $f: B \rightarrow B$ is nonexpanding if $\|f(x)-f(y)\| \leqq\|x-y\|$ for all $x, y \in B$.

Assume that $f: B \rightarrow B$ is nonexpanding. It is not difficult to establish that the set $F$ of fixed points must be convex. Using the con- 